\title{
The Use of Speaking Valves in Children With Tracheostomy Tubes
}

\author{
Weeda Zabih MD MPH, Theresa Holler MD MSc, Faiza Syed RRT, Laurie Russell SLP, \\ Jennifer Allegro SLP, and Reshma Amin MD MSc
}

\author{
Introduction \\ Review of the Literature \\ Research Question \\ Identification and Selection of Studies \\ Research Objectives \\ Charting the Data \\ Summarizing and Reporting Results \\ Results \\ Search Results \\ Characteristics and Level of Evidence of the Selected Studies \\ Eligibility Criteria for Trials of a Speaking Valve \\ Benefits of Speaking Valve Use \\ Discussion \\ Conclusions
}

One-way speaking valves have been successfully used to restore audible meaningful speech in adult patients after tracheostomy tube placement. One-way speaking valves have also been used in pediatric patients after tracheostomy tube placement with promising results. We conducted a scoping review to synthesize and summarize the current evidence on the use of one-way tracheostomy tube speaking valves in the pediatric population to identify knowledge gaps that could inform future research programs and facilitate evidence-based clinical decision making. The Arksey and O'Malley 5-step methodological framework was used for this scoping review. We searched OVID MEDLINE, EMBASE, PubMed, Web of Science, Cumulative Index to Nursing and Allied Health Literature (CINAHL), and Google Scholar to locate articles published between January 1, 1946 and May 26, 2016. Our search resulted in a total of 524 articles. After removing 270 duplicates, we screened 254 abstracts, and 50 articles were identified for full text review. We excluded 38 references. A total of 12 articles met our inclusion criteria. Details of all studies were charted. Application of the Sackett levels of evidence to evaluate the qualitative strength of the evidence provided by the 12 articles selected for study found that 6 studies were level 5,4 were level 4 , and 2 studies were categorized as level 3 evidence. Eligibility criteria for trials of speaking valves were inconsistent across all studies and included a combination of clinical assessment coupled with published indications. Much of the literature has focused on tolerance/successful use of speaking valves in children with a tracheostomy with limited evidence on its impact on verbal communication. Current evidence on the use of speaking valves in children with a tracheostomy, its indication, and its impact on verbal communication is inadequate, mandating further research in this area. Key words: speaking valve; Passy-Muir; tracheotomy speaking valve; tracheostomy speaking valve; swallowing and speaking valve; long-term ventilation speaking valve. [Respir Care 2017;62(12):1594-1601. ( 2017 Daedalus Enterprises] 


\section{Introduction}

A tracheotomy is performed to bypass an upper-airway obstruction, facilitate long-term mechanical ventilation and/or allow for pulmonary clearance. ${ }^{1,2}$ Although principal indications for a pediatric tracheotomy have remained the same over the past decade, more children have tracheostomies for increasingly longer periods of time. ${ }^{1}$

Motor speech production and verbal communication are affected by the presence of a tracheostomy tube in the airway. Ensuring ongoing communication is an important aspect of care for children with tracheostomy tubes. It has previously been shown that the absence of an effective and consistent communication modality has a negative impact on a child's medical, psychological, and social well-being. ${ }^{3}$

The presence of a tracheostomy tube prevents the normal flow of air upward through the vocal folds; the vibration of the vocal folds produces the voice. One-way speaking valves have been successfully used to enable patients to vocalize after tracheostomy tube placement. ${ }^{4}$ A speaking valve is placed on the hub of a tracheostomy tube to redirect air flow upward through the vocal folds during expiration. Criteria for selection of candidates for use of a speaking valve may include: tracheostomy tube size less than two thirds of the tracheal lumen, medical stability, ability to have the tracheostomy cuff deflated without aspiration, patency of airway above the tracheostomy, and absence of thick secretions. ${ }^{5}$

In addition to speech and language acquisition, literature from the adult population suggests additional benefits, including a reduction in aspirations as well as improved secretion management and ease of breathing. ${ }^{6,7}$ One-way speaking valves have been used in children with promising results. However, a current understanding of the extent

Dr Zabih, Ms Syed, and Dr Amin are affiliated with the Division of Respiratory Medicine; Dr Holler is affiliated with the Department of Otolaryngology-Head and Neck Surgery; and Ms Russell and Ms Allegro are affiliated with the Department of Speech Language Pathology, Hospital for Sick Children, Toronto, Canada. Drs Holler and Amin are also affiliated with the University of Toronto, Toronto, Canada.

The authors have disclosed no conflicts of interest.

Supplementary material related to this paper is available at http:// www.rcjournal.com

Correspondence: Reshma Amin MD MSc, Division of Respiratory Medicine, 4539 Hill Wing, Hospital for Sick Children, 555 University Avenue, Toronto, M5G 1X8, Canada. E-mail: Reshma.amin@ sickkids.ca.

DOI: $10.4187 /$ respcare.05599 and scope of the current evidence on the use of speaking valves in children with tracheostomy tubes is lacking.

We conducted a scoping review to synthesize and summarize the current evidence on the use of one-way tracheostomy tube speaking valves in the pediatric population to identify knowledge gaps that could inform future research programs and facilitate evidence-based clinical decision making.

\section{Review of the Literature}

We used the Arksey and O'Malley 5-step methodological framework to conduct our scoping review. ${ }^{8}$ The 5 steps included: (1) identification of the research question; (2) identification of studies relevant to the research question; (3) selection of studies to include in the review; (4) charting, collating, and summarizing the information and data within the included studies; and (5) reporting results of the review.

\section{Research Question}

The research question was formulated by an interprofessional team of clinicians from the Division of Respiratory Medicine, the Department of Otolaryngology-Head and Neck Surgery, and the Departments of Respiratory Therapy and Speech-Language Pathology at the Hospital for Sick Children (Toronto, Canada) based on current knowledge gaps in the use of speaking valves in children. The research question was as follows: What is the scope of existing literature to support the use of speaking valves in children with tracheostomy tubes?

\section{Identification and Selection of Studies}

We searched the following electronic databases from January 1, 1946 to May 26, 2016: OVID MEDLINE, EMBASE, PubMed, Web of Science, Cumulative Index to Nursing and Allied Health Literature (CINAHL), and Google Scholar. We also hand-searched key websites and existing organizations and networks, which included the Canadian, American, and European Respiratory Society websites as well as the Passy-Muir website.

Search terms were developed in consultation with an experienced information specialist. All studies that described a pediatric population (age $0-18$ y) using a oneway tracheostomy speaking valve were included. Search terms such as "speaking valve," "Passy-Muir," "tracheotomy speaking valve," "tracheostomy speaking valve," "swallowing and speaking valve" and "long-term ventilation speaking valve" were used to locate studies relevant to our review.

We included all types of studies that described children with a tracheostomy using a speaking valve. Observational 
studies, randomized controlled trials, before-and-after (experimental) studies, retrospective studies, case series, and qualitative studies were included. We excluded single case reports, review articles, book excerpts, commentaries, editorials, opinion papers, and studies that were written in languages other than English because they did not fall within the 5 levels of evidence as per the Sackett levels of evidence scale (see below). ${ }^{9,10}$ We used an EndNote X4 (Clarivate Analytics, Philadelphia, Pennsylvania) library for maintaining and managing our records.

Using a predesigned screening tool, 2 authors (RA and WZ) independently examined study titles and abstracts to identify eligible studies. For studies that were deemed potentially eligible, full text articles were obtained and reviewed by both authors for eligibility. Disagreements were resolved through discussion with a third author (TH).

\section{Research Objectives}

We had 2 main research objectives for this scoping review. Research objective 1 was to report the eligibility criteria in the literature for the use of speaking valves in children with tracheostomies. Research objective 2 was to summarize the reported benefits from the use of a speaking valve.

\section{Charting the Data}

The research team collectively developed a standardized charting form that included all of the variables relevant to the research question. The charting form was iteratively updated as needed. Using the charting form, each author (RA and WZ) independently abstracted the information from each article included. This information was then verified and checked for accuracy by a third author (TH). For each article included in this review, the charting form was utilized to collect the following data points: type of study, authors, year of publication, study setting, sample size, country where the study was conducted, age range of study participants, primary diagnosis and comorbidities of study participants, inclusion and exclusion criteria used for trials of the speaking valve, and the study outcomes.

\section{Summarizing and Reporting Results}

Summary tables were generated to report the counts and proportions of the study and cohort characteristics. We used the Sackett levels of evidence scale to evaluate the quality of each study. ${ }^{9,10}$ This tool ranks articles from 1 (strongest evidence) to 5 (weakest evidence) based on the type of research and probability of bias. The levels of evidence according to Sackett are as follows: level 1, randomized control trials with clear-cut results; level 2, small

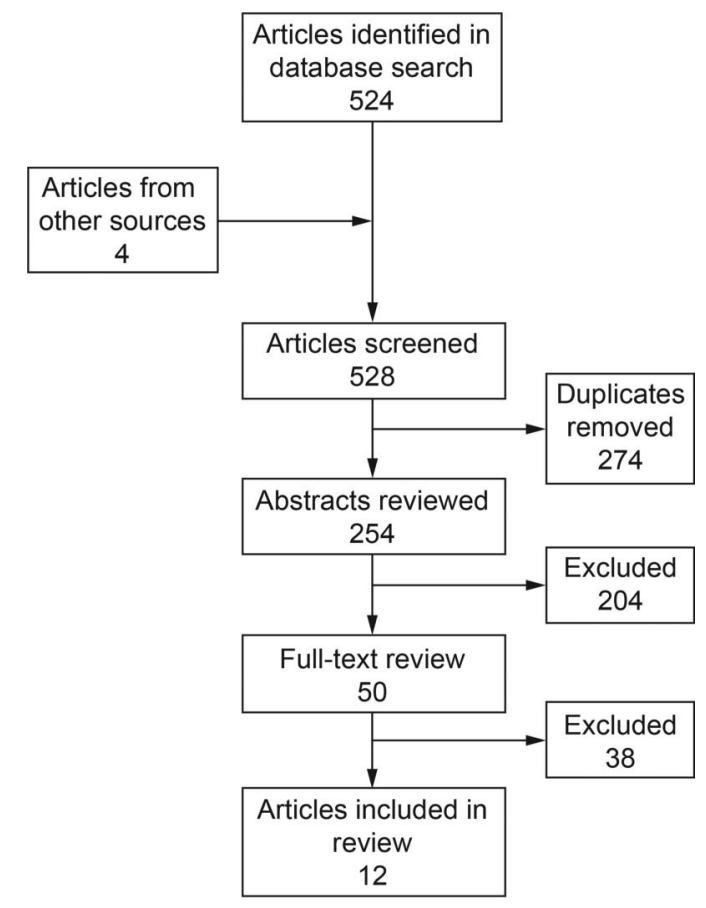

Fig. 1. Flow chart.

randomized controlled trials with unclear results; level 3, cohort and case-control studies; level 4, historical cohort or case-control studies; level 5, case series studies with no controls. Information such as type of study, population, and research methods were scanned for each article to determine the level of evidence. A qualitative analytic method was used to identify, analyze, and report patterns in the data. For each article, characteristics of the study participants, selection of candidates for speaking valve trials, and study outcomes were reported.

\section{Results}

\section{Search Results}

As delineated in Figure 1, our search resulted in a total of 524 articles; we excluded 270 duplicates. Our hand search of journals and relevant websites resulted in 4 additional articles. We screened 254 abstracts and identified 50 references for full text review. Of these 50 references, 38 were excluded for reasons of focus (ie, not about speaking valves) and population studied (ie, not pediatric). A total of 12 articles met our inclusion criteria.

\section{Characteristics and Level of Evidence of the Selected Studies}

For the 12 studies included in this review, the sample size ranged from 2 to 64 children. Nine studies $(75 \%)$ were 
conducted in the United States, one $(8.3 \%)$ in the United Kingdom, one (8.3\%) in Australia, and one $(8.3 \%$ ) in Thailand. Study designs included prospective cohort $(16.6 \%$, no. $=2)$, prospective case control $(16.6 \%$, no. $=2)$, retrospective cohorts $(16.6 \%, n=2)$, and case series $(50 \%$, no. $=6)$. Six studies $(50 \%)$ were categorized as level 5 evidence. Four studies (33.3\%) were level 4 evidence, and 2 studies (16.6\%) were level 3 evidence. Two studies $(16.6 \%)$ reported on the use of a speaking valve in an in-patient care setting, one (8.3\%) in the ICU, and $2(16.6 \%)$ in the NICU. The remaining studies (58\%) reported the use of a speaking valve in an out-patient setting. See Table 1 and Table 2 for a summary of the study characteristics of the included studies that were graded using the Sackett levels of evidence.

Table 1 provides a summary of subject characteristics of the studies. Two studies (16.6\%) reported on the use of a drilled speaking valve (a modified type of speaking valve with up to 2 holes, each $1.6 \mathrm{~mm}$ ). The population of children included in each study for trials of a speaking valve was heterogeneous with regard to primary diagnosis, reasons for tracheotomy, and comorbidities. Most of the studies included children with more than one comorbidity and multiple diagnoses, including congenital and chromosomal abnormalities, mental, neurologic, musculoskeletal, and respiratory disorders. Key reported indications for tracheotomy included upper-airway obstruction, pulmonary clearance, and prolonged mechanical ventilation.

Two studies ${ }^{4,16}$ included a total of 12 children who had tracheostomies for upper-airway obstruction. Five studies ${ }^{4,12-14,20}$ included a total of 33 children with a tracheostomy for mechanical ventilation. The remaining 5 studies ${ }^{11,17-19,21}$ included 162 children who had varied indications for tracheostomy. Specifically, 82 had a tracheostomy for upper-airway obstruction and laryngeal abnormalities, 54 required a tracheostomy for mechanical ventilation, and 26 children had a tracheostomy for pulmonary clearance (see Table 1 ).

\section{Eligibility Criteria for Trials of a Speaking Valve}

The majority of studies reported on the use of one of 2 main strategies to determine eligibility for a speaking valve trial: (1) clinical assessment by a team of experts or (2) indications listed from a speaking valve manufacturer as a general guide and population-specific criteria. The clinical team of experts included pediatric otolaryngology head and neck surgery, speech and language pathology, pediatric pulmonology/general pediatrics, and pediatric respiratory therapy (see Table 1, bottom). Seven studies (58.3\%) reported a team approach to assessing children before a speaking valve trial. Cho Lieu et $\mathrm{al}^{11}$ included children assessed by the tracheal airway communication team. No specific inclusion criteria were reported. Four stud-
Table 1. Characteristics of Studies Included

\begin{tabular}{|c|c|}
\hline Characteristics & no. $(\%)$ \\
\hline \multicolumn{2}{|l|}{ Study characteristics } \\
\hline \multicolumn{2}{|l|}{ Country } \\
\hline United States & $9(75.0)$ \\
\hline United Kingdom & $1(8.3)$ \\
\hline Australia & $1(8.3)$ \\
\hline Thailand & $1(8.3)$ \\
\hline \multicolumn{2}{|l|}{ Study design } \\
\hline Prospective cohort & $2(16.6)$ \\
\hline Prospective case control & $2(16.6)$ \\
\hline Retrospective cohorts & $2(16.6)$ \\
\hline Case series & $6(50.0)$ \\
\hline \multicolumn{2}{|l|}{ Study setting } \\
\hline In-patient & $2(16.6)$ \\
\hline $\mathrm{ICU}$ & $1(8.3)$ \\
\hline NICU & $2(16.6)$ \\
\hline Out-patient & $7(58.0)$ \\
\hline \multicolumn{2}{|l|}{ Sample size } \\
\hline $1-10$ & $5(41.6)$ \\
\hline $11-30$ & $5(41.6)$ \\
\hline $31-65$ & $2(16.6)$ \\
\hline \multicolumn{2}{|l|}{ Sackett level of evidence } \\
\hline Level 5 & $6(50.0)$ \\
\hline Level 4 & $4(33.3)$ \\
\hline Level 3 & $2(16.6)$ \\
\hline Levels 1 and 2 & $0(0.0)$ \\
\hline \multicolumn{2}{|l|}{ Cohort characteristics } \\
\hline \multicolumn{2}{|l|}{ Type of speaking valve used } \\
\hline Passy-Muir & $9(75.0)$ \\
\hline Shiley + Passy-Muir & $1(8.3)$ \\
\hline Drilled Passy-Muir & $2(16.6)$ \\
\hline Total number of children included in 12 studies & $230(100)$ \\
\hline Upper-airway obstruction & $111(48.2)$ \\
\hline Long-term ventilation & $84(36.5)$ \\
\hline Pulmonary clearance & $35(15.2)$ \\
\hline \multicolumn{2}{|l|}{ Eligibility criteria for speaking valve trial } \\
\hline Team assessment and added criteria & $7(58.3)$ \\
\hline $\begin{array}{l}\text { Passy-Muir valve manufacturer guidelines } \\
\text { and added inclusion/exclusion criteria }\end{array}$ & $5(33.3)$ \\
\hline \multicolumn{2}{|l|}{ Reported benefits } \\
\hline Success/tolerance of use (awake hours) & $10(83.0)$ \\
\hline Safety of use during sleep & $1(8.3)$ \\
\hline Improved communication & $8(66.6)$ \\
\hline Secretion management/aspiration & $3(25.0)$ \\
\hline Improved swallowing & $2(16.6)$ \\
\hline Improved cough & $1(8.3)$ \\
\hline Ease of breathing & $1(8.3)$ \\
\hline
\end{tabular}

ies $^{12,15,20,21}$ reported the involvement of a team and a teambased protocol. In addition, these studies reported inclusion criteria for trials of a speaking valve. Gereau et al ${ }^{14}$ reported selection of children based on speaking valve manufacturer recommendations and additional inclusion criteria introduced by a team of experts. Buckland et $\mathrm{al}^{16}$ included children for trials of a drilled speaking valve based 
Pediatric Use of Speaking Valves With Tracheostomy

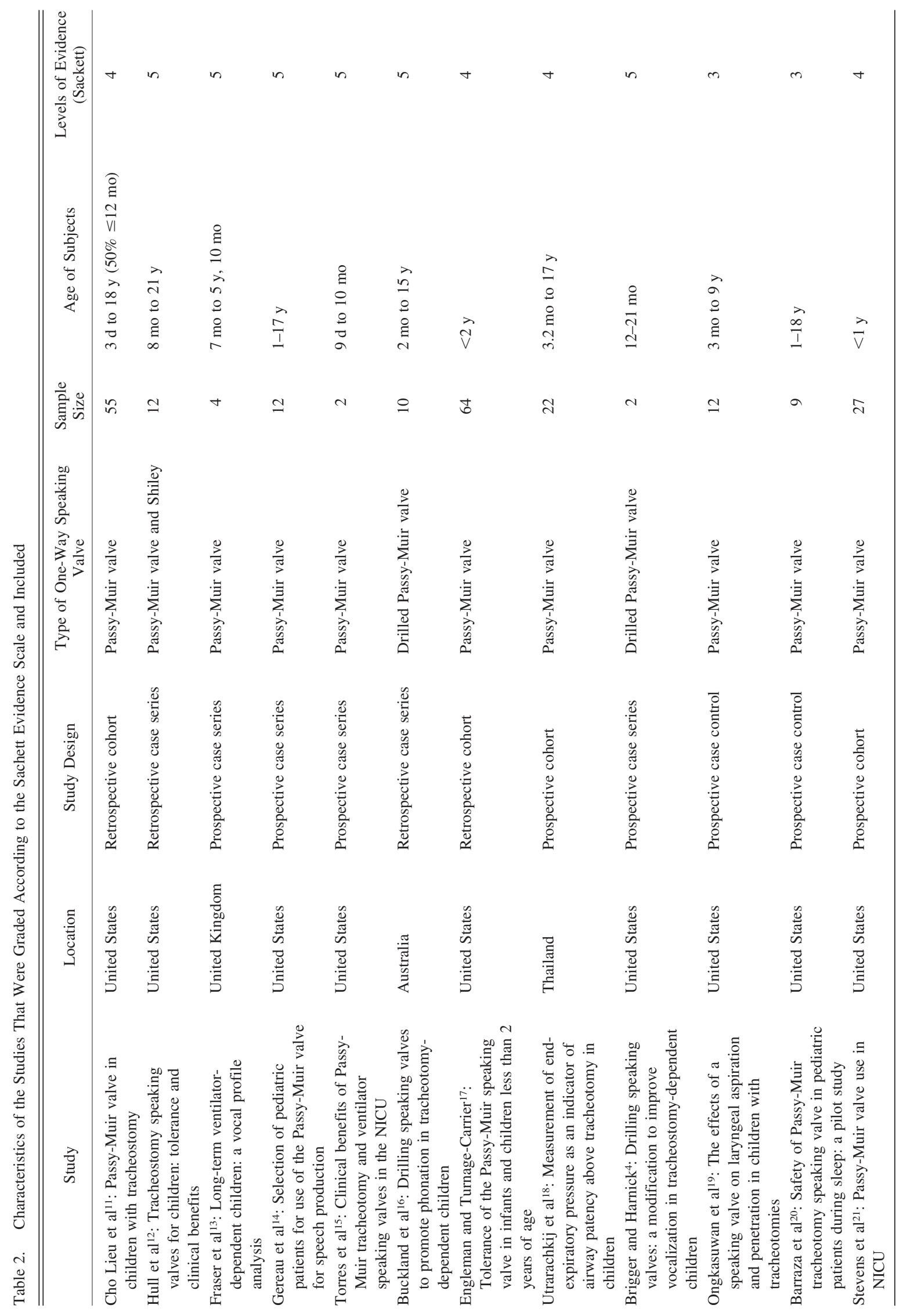


on a protocol and team assessment. In addition, authors also reported a decision making algorithm that specified each team member's role in the assessment process.

Four studies, 13,17,18 (33.3\%) used speaking valve manufacturer indications as a general guide and reported additional inclusion/exclusion criteria. One study ${ }^{19}$ (8.3\%) did not report any inclusion/exclusion criteria. Supplementary Table 1 provides details on the inclusion criteria for speaking valve candidacy (see the supplementary table at http://www.rcjournal.com).

\section{Benefits of Speaking Valve Use}

The reported benefits of a speaking valve are summarized in Table 1 . The most commonly reported study outcome (primary and secondary study outcome) was tolerance/successful use of a speaking valve, reported in 10 studies. ${ }^{4,11,12,14-18,20,21}$ Of these 10 studies, only $4^{12,15-17}$ studied tolerance/successful use of a speaking valve as a primary outcome. These 4 studies $^{11,12,17,20}$ defined tolerance/successful use of a speaking valve during awake hours as stable vital signs, stable oxygen saturation, and absence of symptoms of intolerance (eg, agitation, anxiety, skin color change, coughing/gagging) from a baseline before trials of a speaking valve were introduced. Ninety-three of 103 subjects from these studies tolerated the speaking valve if trialed. Five ${ }^{14,15,18,19,21}$ (of a total of 10) studies reported tolerance and successful use of a speaking valve during awake hours as a secondary outcome but did not define tolerance or success. One study ${ }^{20}$ (of a total of 10) reported safety of a speaking valve during sleep as a primary outcome, defined as stable vital signs and gas exchange (oxygen saturation and end-tidal $\left.\mathrm{CO}_{2}\right) 1$ night before trials of a speaking valve. A speaking valve while asleep was safely used in all 7 children it was trialed on. ${ }^{20}$

Verbal communication was reported in 8 studies.,411,15-17,12-14 Only one study ${ }^{13}$ reported communication (vocal function with speaking valve) as a primary outcome in a case series. In this study, 12 parameters of communication were selected for assessment by 3 trained speech therapists and rated as normal and abnormal. Parameters included: modal voice, phonation type, pitch, loudness, breath support, and voice continuity. The study recorded spontaneous speech in older children and babbling in infants and those in the prelinguistic developmental stage. Two subjects scored 9 and 10 out of 12 , and the other 2 had a poor score ( 3 and 4 out of 12). ${ }^{13}$ In all other studies, ${ }^{4,11,12,14-17}$ communication was assessed clinically using different nonspecific terms and studied as secondary outcomes. Audible crying, nonspecific vocalization, word approximation, single words, short phrases, making noises, and production of voice were terms used to report communication with a speaking valve. For studies that included different age groups, no age-specific milestone was used to evaluate communication and speech. Two studies ${ }^{4,16}$ reported communication with a drilled speaking valve in children with upper-airway obstruction. Communication was defined as "production of sustained voice for $\geq 2$ seconds" by Buckland et $\mathrm{al}^{16}$ and reported as production of voice based on clinical assessment by Brigger et al. ${ }^{4}$ Overall, audible phonation was feasible in 107 of 144 pediatric subjects between $3 \mathrm{~d}$ and 12 months of age across 7 studies of speaking valve use in children with a tracheostomy. 4,11-16

Other benefits of a speaking valve, including improved secretion management; better cough management; and/or improved swallowing; ease of breathing; and reduced aspiration were reported in 6 studies $^{11,12,15,19,21}$ (50\%). Only one article ${ }^{19}$ studied reduced aspiration with a speaking valve as a primary outcome. This study used a scoring system and fluoroscopy to define and report reduced laryngeal aspiration and improved swallowing. A speaking valve was shown to have no effect on aspiration in any of the 12 pediatric subjects between the ages of 3 months and 9 y. ${ }^{19}$ Other secondary benefits, such as improved secretion management, better cough management, ease of swallowing, and ease of breathing, were reported by caregivers or assessed by clinicians ${ }^{11,12,15,21}$ but were not defined a priori. Additional details of reported benefits and key findings for each study are given in Table 3 .

\section{Discussion}

The main aim of our review was to summarize and synthesize the current body of evidence related to speaking valve use in children with tracheostomy tubes. Overall, there is a dearth of strong evidence (level 1 and 2 studies) to support the use of one-way speaking valves in the pediatric population. A Passy-Muir valve is the only oneway speaking valve that has been studied in children. The current level of evidence is inadequate to optimally guide clinical decision making for speaking valve use in children who have a tracheostomy, and there is a need for welldesigned randomized controlled trials and cohort studies in this field. Furthermore, there is a need to develop clinical practice guidelines using multidisciplinary panels of experts to provide pragmatic guidance for clinicians, given the current paucity of data in this field.

Most of the current literature has focused on tolerance of speaking valves. The literature does not provide sufficient evidence regarding the impact of a speaking valve on verbal communication in children. Communication is an important aspect of early childhood development, and this area needs to be further studied and explored. Critical periods of speech and language acquisition and age-specific communication milestones early in life for children with tracheostomy tubes should be taken into consideration when designing future studies. 


\section{Pediatric Use of Speaking Valves With Tracheostomy}

Table 3. Study Outcomes and Key Findings of the Studies Graded According to the Sackett Evidence Scale

\begin{tabular}{l}
\hline Study \\
$\begin{array}{l}\text { Cho Lieu et al }{ }^{11} \text { : Passy-Muir valve in children } \\
\text { tracheostomy }\end{array}$ \\
$\begin{array}{c}\text { Hull et al }{ }^{12} \text { : Tracheostomy speaking valves for } \\
\text { children: tolerance and clinical benefits }\end{array}$
\end{tabular}
children: tolerance and clinical benefits

Fraser et al ${ }^{13}$ : Long-term ventilator-dependent children: a vocal profile analysis

Gereau et al ${ }^{14}$ : Selection of pediatric patients for use of the Passy-Muir valve for speech production

Torres et al ${ }^{15}$ : Clinical benefits of Passy-Muir tracheotomy and ventilator speaking valves in the NICU

Buckland et al ${ }^{16}$ : Drilling speaking valves to promote phonation in tracheotomy-dependent children

Engleman and Turnage-Carrier ${ }^{17}$ : Tolerance of the Passy-Muir speaking valve in infants and children less than 2 years of age

Utrarachkij et al ${ }^{18}$ : Measurement of end-expiratory pressure as an indicator of airway patency above tracheotomy in children

Brigger and Harnick ${ }^{4}$ : Drilling speaking valves: a modification to improve vocalization in tracheostomy dependent children

Ongkasuwan et $\mathrm{al}^{19}$ : The effects of a speaking valve on laryngeal aspiration and penetration in children with tracheotomies

Barraza et $\mathrm{al}^{20}$ : Safety of Passy-Muir tracheotomy speaking valve in pediatric patients during sleep: a pilot study

Stevens et $\mathrm{al}^{21}$ : Passy-Muir valve use in NICU

Defined and Reported Outcomes

Successful use of Passy-Muir valve defined as pass/conditional pass (stable heart rate, breathing frequency, oxygen saturation, skin color, and activity level, ability to adapt to an oral exhalation pattern, lack of coughing/gagging, stable behavior and mood)

Decannulation status (time with tracheostomy in months)

Tolerance of tracheotomy speaking valve (minimal anxiety, $\mathrm{O}_{2}$ saturation $\geq 95 \%$, stable heart rate and breathing frequency for duration of trial)

Phonation with speaking (audible crying, nonspecific vocalizations, word approximations, single words, and shor phrases)

Secondary benefits of valve reported (coughing management, secretion management, swallowing/feeding and oxygenation (clinically observed)

Vocal function with Passy-Muir valve (phonation, pitch, loudness, breath support, and continuity measured using an auditory perceptual rating scheme)

Vocalization/speech production and successful use of Passy-Muir valve

Communication (crying, making noise)

Secretion management (reduced secretion and aspiration, ease of breathing)

Ease of swallowing

Tolerance of drilled Passy-Muir valve (wearing the speaking valve for $\geq 2$-h periods within 2 weeks of valve introduction, transpulmonary pressure $\leq 10 \mathrm{~cm} \mathrm{H}_{2} \mathrm{O}$ on passive manometry, no significant changes in arterial oxygenation, heart rate, or breathing during trials)

Phonation with drilled Passy-Muir valve (production of sustained voice for $\geq 2 \mathrm{~s}$ )

Tolerance of Passy-Muir Valve $\left(\mathrm{O}_{2}\right.$ saturation of $\geq 88 \%$, stable breathing frequency, heart rate, no agitation and no change in color, no increase in respiratory effort from baseline)

Vocalization with Passy-Muir valve (clinically assessed as production of voice)

Tolerance of Passy-Muir valve (stable heart rate, breathing frequency, and $\mathrm{O}_{2}$ saturation for $5 \mathrm{~min}$ after speaking valve introduction)

Tolerance of Passy-Muir valve and drilled Passy-Muir valve (trans-tracheal pressure of $<10$, lack of respiratory distress assessed clinically)

Vocalization with drilled Passy-Muir valve (production of voice assessed clinically)

Laryngeal aspiration with Passy-Muir valve (decrease in laryngeal aspiration and penetration measured with Rosenbek 8-point scale and barium swallow)

Improved swallowing with Passy-Muir valve (decreased piriform sinus residue)

Safety of Passy-Muir valve during sleep (stable heart rate, breathing frequency, oxygen saturation, and end-tidal carbon dioxide compared with baseline one night before placing the Passy-Muir valve)

Safety of Passy-Muir valve use (lack of complications assessed clinically)

\section{Key Findings Reported}

52 children tolerated use of speaking valve

10 children tolerated speaking valve and achieved phonation

Minimal to no improvement in coughing, secretion, and swallowing

1 of 4 children on mechanical ventilation had normal speech

12 children could successfully use a speaking valve and vocalize

2 children on mechanical ventilation could tolerate the valve and produced voice

8 children tolerated drilled speaking valve

5 children could phonate using the drilled valve

24 tolerated a speaking valve use

23 produced vocalization

13 children tolerated use of a speaking valve

2 children tolerated a drilled speaking valve and could communicate

12 children showed no improvement in aspiration, but improvement in swallowing was reported

7 children safely used a speaking valve during sleep

19 children safely used a speaking valve
In addition, there is minimal anecdotal evidence from case series and small studies regarding the secondary benefits of a speaking valve, including better secretion and cough management, improved swallowing, increased ease of breathing, and reduced aspiration. Considering the medical vulnerability of children living with a tracheostomy in the home milieu who are prone to respiration-related complications, addressing this gap in the literature may be an opportunity to reduce morbidity and potentially contribute to a reduction in mortality of these children.
The population of children included in the majority of the studies was heterogeneous with regard to primary diagnosis, comorbidities, and indications for tracheotomy with collective results reported. Three main reasons for tracheotomy were upper-airway obstruction, the need for long-term ventilation, and pulmonary toilet. Because these characteristics are likely to impact the use of speaking valves and the outcomes, future studies are needed that are sufficiently powered to cogently study these subgroups of children. Evidence from such studies will result in a sci- 


\section{Pediatric Use of Speaking Valves With Tracheostomy}

entifically based general consensus on indications for use of a speaking valve that could be used consistently by health professionals and researchers in the future.

\section{Conclusions}

This review documents the lack of adequate qualitative and quantitative evidence on the use of a speaking valve in the pediatric population ( $<18$ y old) with tracheostomy tubes. Verbal communication, although a crucial aspect of care of children with a tracheostomy, has not been adequately explored in the current scientific literature. There is a need for pragmatic and interventional studies that can better inform clinical practice in the future.

\section{REFERENCES}

1. Lawrason A, Kavanagh K. Pediatric tracheotomy: are the indications changing? Int J Pediatr Otorhinolaryngol 2013;77(6):922-925.

2. Kremer B, Botos-Kremer AI, Eckel HE, Schlöndorff G. Indications, complications, and surgical techniques for pediatric tracheostomies-an update. J Pediatr Surg 2002;37(11):1556-1562.

3. Baker BM, Blackwell PB. Adult and infant vocalization: speaking valves used in individuals with tracheostomy and ventilator dependency. J Ky Med Assoc 2001;99(5):193-203.

4. Brigger MT, Hartnick CJ. Drilling speaking valves: a modification to improve vocalization in tracheostomy dependent children. Laryngoscope 2009;119(1):176-179.

5. Sherman JM, Davis S, Albamonte-Petrick S, Chatburn RL, Fitton C, Green $\mathrm{C}$, et al. Care of the child with a chronic tracheostomy: this official statement of the American Thoracic Society was adopted by the ATS Board of Directors, July 1999. Am J Respir Crit Care Med 2000;161(1):297-308.

6. Adam SI, Srinet P, Aronberg RM, Rosenberg G, Leder SB. Verbal communication with the Blom low profile and Passy-Muir one-way tracheotomy tube speaking valves. J Commun Disord 2015;56:4046.

7. Bultsma R, Koopmans M, Kuiper M, Egbers P. Ability to speak in ventilator-dependent tracheostomized ICU patients. Crit Care 2014; 18(Suppl 1):P323.
8. Arksey H, O'Malley L. Scoping studies: towards a methodological framework. Int J Soc Res Methodol 2005;8(1):19-32.

9. Sackett D SS, Richardson W. Evidence-based medicine: how to practice and teach EBM. Edinburgh: Churchill Livingstone; 2001.

10. Sackett D, Rosendberg W, Haynes R. Evidence-based medicine: how to practice and teach EBM, 2nd edition. Edinburgh, Scotland: Churchill Livingston; 2003.

11. Cho Lieu JE, Muntz HR, Prater D, Blount Stahl M. Passy-Muir valve in children with tracheotomy. Int J Pediatr Otorhinolaryngol 1999; 50(3):197-203.

12. Hull EM, Dumas HM, Crowley RA, Kharasch VS. Tracheostomy speaking valves for children: tolerance and clinical benefits. Pediatr Rehabil 2005;8(3):214-219.

13. Fraser J, Pengilly A, Mok Q. Long-term ventilator-dependent children: a vocal profile analysis. Pediatr Rehabil 1998;2(2):71-75.

14. Gereau SA, Navarro GC, Cluterio B, Mullan E, Bassila M, Ruben RJ. Selection of pediatric patients for use of the Passy-Muir valve for speech production. Int J Pediatr Otorhinolaryngol 1996;35(1):11-17.

15. Torres LY, Sirbegovic DJ. Clinical benefits of the Passy-Muir tracheostomy and ventilator speaking valves in the NICU. Neonatal Intensive Care. 2004;17(4):20-23.

16. Buckland A, Jackson L, Ilich T, Lipscombe J, Jones G, Vijayasekaran S. Drilling speaking valves to promote phonation in tracheostomy-dependent children. Laryngoscope 2012;122(10):2316-2322.

17. Engleman SG, Turnage-Carrier C. Tolerance of the Passy-Muir speaking valve in infants and children less than 2 years of age. Pediatr Nurs 1997;23(6):571-573.

18. Utrarachkij J, Pongsasnongkul J, Preutthipan A, Chantarojanasri T. Measurement of end-expiratory pressure as an indicator of airway patentcy above tracheostomy in children. J Med Assoc Thai 2005; 88(7):928-933

19. Ongkasuwan J, Turk CL, Rappazzo CA, Lavergne KA, Smith EO, Friedman EM. The effect of a speaking valve on laryngeal aspiration and penetration in children with tracheotomies. Laryngoscope 2014; 124(6): 1469-1474.

20. Barraza G, Halaby C, Islam S, Gutekunst W, Simpser E, Pirzada M. Safety of Passy-Muir tracheostomy speaking valve in pediatric patients during sleep: a pilot study. Chest Conference meeting abstract; 2013. doi: $10.1378 /$ chest. 1692327

21. Stevens M, Finch J, Justice L, Geiger E. Use of the Passy-Muir valve in the neonatal intensive care unit. Neonatal Intensive Care 2011; 24(7):22-23. 